\title{
Haplotype and linkage disequilibrium analysis to characterise a region in the calcium channel gene CACNA1A associated with idiopathic generalised epilepsy
}

Barry Chioza $^{1}$, Abena Osei-Lah ${ }^{2}$, Lina Nashef ${ }^{2,3}$, Blanca Suarez-Merino ${ }^{4}$, Hazel Wilkie ${ }^{1}$, Pak Sham ${ }^{1,5}$, Jo Knight ${ }^{5}$, Philip Asherson ${ }^{5}$ and Andrew J Makoff*,1,5

${ }^{1}$ Department of Psychological Medicine, Institute of Psychiatry, London SE5 8AF, UK; ${ }^{2}$ Department of Neuroscience, Kings' College Hospital, London SE5 9RS, UK; ${ }^{3}$ Department of Neurology, Kent and Canterbury Hospital, Canterbury CT1 3NG, UK; ${ }^{4}$ Institute of Neurology, London WC1N 3BG, UK; ${ }^{5}$ Social Genetic and Developmental Psychiatry Research Centre, Institute of Psychiatry, London SE5 8AF, UK

Idiopathic generalised epilepsy (IGE) is a common form of epilepsy, including several defined and overlapping syndromes, and likely to be due to the combined actions of mutations in several genes. In a recent study we investigated the calcium channel gene CACNA1A for involvement in IGE, unselected for syndrome, by means of association studies using several polymorphisms within the gene. We reported a highly significant case/control association with a silent single nucleotide polymorphism (SNP) in exon 8 that we confirmed by within-family analyses. In this present study we screened the gene for novel SNPs within $25 \mathrm{~kb}$ of exon $\mathbf{8}$, which have enabled us to define the critical region of CACNA1A in predisposing to IGE. Several intronic SNPs were identified and three, within $1.5 \mathrm{~kb}$ of exon 8 and in strong linkage disequilibrium with each other and with the original SNP, were significantly associated with IGE $(P=0.00029, P=0.0015$ and $P=0.010)$. The associations were not limited to an IGE syndrome or other subgroup. Another SNP, $25 \mathrm{~kb}$ away, in intron 6 was also significantly associated with IGE $(P=0.0057)$ but is not in linkage disequilibrium with the SNPs around exon 8. Haplotype predictions revealed even more significant associations (3-marker haplotype: $P<10^{-6}$ ). Logistic regression showed that all the data can be explained by two of the SNPs, which is consistent with two functionally significant variants being responsible for all five associations, although a single variant cannot be excluded. The functionally significant variant(s) are unlikely to be exonic and suggests an effect on expression or alternative splicing. European Journal of Human Genetics (2002) 10, 857-864. doi:10.1038/sj.ejhg.5200896

Keywords: CACNA1A; voltage-gated calcium channel; idiopathic generalised epilepsy (IGE); linkage disequilibrium; SNPs; haplotype

\section{Introduction}

Idiopathic generalised epilepsy (IGE) is a common disorder, with a cumulative incidence in the general population of

\footnotetext{
*Correspondence: Dr AJ Makoff; Department of Psychological Medicine, Institute of Psychiatry, Denmark Hill, London SE5 8AF, UK. Tel: 44-(0)20-7848-0638; Fax: 44-(0)20-7848 0051;

E-mail: a.makoff@iop.kcl.ac.uk

Received 22 March 2002; revised 15 August 2002; accepted 26 August 2002
}

$0.7 \%{ }^{1}$ IGE usually presents in childhood or adolescence. Seizure types include generalised tonic-clonic seizures (GTCS), myoclonic seizures and/or absences. Generalised spike waves or polyspike waves are observed on electroencephalography. A photoparoxysmal response may be observed. There are no associated neurological abnormalities or structural brain damage. Twin studies demonstrate the importance of genetic factors in IGE susceptibility, with concordance rates for monozygotic 
twin pairs far higher than for dizygotic twin pairs. ${ }^{2,3}$ Common forms of IGE show a complex pattern of inheritance, which, in keeping with other common genetic disorders, is expected to result from the action of a few or many genes of small to moderate effect. IGE includes many common syndromes such as juvenile myoclonic epilepsy (JME), childhood absence epilepsy (CAE) and juvenile absence epilepsy (JAE). ${ }^{4}$ There is considerable overlap between these syndromes and it is not always possible to subclassify cases. More than one IGE syndrome is frequently observed within families., ${ }^{5,6}$ In a twin study, out of 20 monozygotic pairs all 13 that were clinically concordant had the same syndrome of IGE in both twins. ${ }^{7}$ By comparison, there were five out of 21 clinically concordant dizygotic pairs, of which only one had the same syndrome. These observations suggest that several combinations of mutations cause IGE but only certain combinations are syndrome specific.

Linkage studies have proved very successful in identifying causative mutations in Mendelian disorders. However, they have been much less useful in complex disorders where possession of a susceptibility mutation is neither necessary nor sufficient to cause the disease. Very large sample sizes are required to achieve the necessary power to detect linkage with genes of such disorders, and as a result linkage studies are often underpowered. Many susceptibility mutations are likely to be common and association studies provide far more power to detect common mutations of small to moderate effect with sample sizes of a few hundred unrelated affected individuals. ${ }^{8,9}$ Although case-control studies are vulnerable to population stratification artefacts, within-family tests of association can be used which are robust to such stratification.

Mouse mutants, such as tottering, lethargic, and ducky, share a number of features with human IGE and have served as useful models for absence epilepsy. These include age-related presentations, the presence of generalised spike wave discharges, involvement of thalamo-cortical circuits and responses to pharmacological agents. ${ }^{10-12}$ Interestingly, these models are now known to be due to mutations in voltage-gated calcium channel subunits, all of which appear to be involved in P/Q-type channels: tottering (Cacna1a), lethargic (Cacnb4) and ducky (Cacna2d2). ${ }^{13-15}$ To date, four spontaneous recessive mutations in the mouse gene Cacna1a encoding the $\alpha_{1 \mathrm{~A}}$ subunit in P/Q-type channels have been described: tottering, rolling Nagoya, leaner and rocker. ${ }^{13,16,17}$ All include ataxia, while tottering and the more severe phenotype, leaner, also exhibit motor seizures and absence type seizures. Mutations in the human gene CACNA1A result in episodic ataxia 2 (EA-2), spinocerebellar ataxia type 6 (SCA6) and familial hemiplegic migraine (FHM). ${ }^{18,19}$ One ataxic child with absence seizures was shown to have a mutation in CACNA1A. ${ }^{20}$ Most of CACNA1A has been sequenced and has 47 exons extending over approximately $300 \mathrm{~kb}$ of DNA. ${ }^{18}$
Recently we reported an association study of IGE using five polymorphisms within CACNA1A in over 200 cases with a broad IGE phenotype. ${ }^{21}$ Comparison of the allele frequencies between probands and controls for a single nucleotide polymorphism (SNP) in exon 8 provided evidence for significant association with IGE $(P=0.0003$, $\mathrm{OR}=1.7$ ). Haplotype-based haplotype relative risk (HHRR) and transmission disequilibrium test (TDT), robust to possible population stratification, confirmed this result and supporting evidence came from an association with a simple sequence repeat polymorphism in intron 7 , D19S1150 $(P=0.032)$. Other SNPs in exons 6, 16 and 20 were not associated with IGE. In a previous independent study of IGE families, a CAG repeat polymorphism in exon 47 was not associated with IGE. ${ }^{22}$ Here, using an enlargement of the same sample used in our earlier study, ${ }^{21}$ we report on an investigation of the region flanking the associated SNP in exon 8, the first step in delineating the region of CACNA1A containing a common functional variant(s) associated with IGE.

\section{Materials and methods \\ Sample}

The study included 232 probands, 234 controls and both parents from a subset of approximately half the probands. This is the same sample used for our previous study ${ }^{21}$ but with increased numbers. IGE probands were identified via the EEG Department and epilepsy clinics at Kent and Canterbury Hospital. DNA was obtained from Caucasian subjects via cheek swabs or blood. Probands with epilepsy were selected with generalised spike/wave (GSW) on EEG, normal background rhythm and a consistent clinical history. Control samples were collected from the same hospital from unrelated individuals in the same ethnic group but with no known history of epilepsy/blackouts. At the time of sample collection, the mean age of the probands was 23 years with $60 \%$ female and the mean age of the controls was 41 years with $59 \%$ female. Detailed clinical characterisation has been compiled by examination of case notes and EEG reports by A Osei-Lah and L Nashef and, in some cases, clinical assessment by $\mathrm{L}$ Nashef. Information on seizure types, pattern and age of onset, EEG results, imaging data where performed, family history and response to treatment was extracted. Classification by ILAE IGE syndrome was made according to strict criteria. Where syndrome classification was not possible, broad or overlap categories were used. Mean age of onset of the epilepsy was 11.7 , with $85 \%$ presenting between age 3 and 20. Information was available on seizure types in over $92 \%$ of probands: $58 \%$ had absences, $77 \%$ generalised tonic-clonic seizures (GTCS), and $23 \%$ myoclonus. A previous history of febrile convulsions was present in $14 \%$ and $34 \%$ had photoparoxysmal response reported on EEG. Classification by ILAE syndrome ${ }^{23}$ on $78 \%$ of the sample was as 
follows: idiopathic absence epilepsy (IAE-absences with or without GTCS but without myoclonus) 50\% (CAE 21\%, JAE 16\%, other 13\%), JME $17 \%$ (including $7 \%$ with prominent absences) and Epilepsy with GTCS only $26 \%$. Other syndromes or overlap syndromes accounted for the remaining $7 \%$.

\section{Denaturing high performance liquid chromatography (DHPLC) analysis}

DHPLC analysis was performed using the Transgenomic WAVE DNA Analysis System. Chromosomal regions investigated for SNPs were amplified by PCR to give overlapping amplicons of approximately $500 \mathrm{bp}$ in length, the optimum size for both DHPLC and automated sequencing. The amplicon sequence was entered into the WAVEMAKER software, which predicts temperature and gradient conditions that resolve heteroduplexes on the WAVE system. Between $5-15 \mu$ l of crude PCR product was loaded on a DNASep column (Transgenomic, Santa Clara, CA, USA). The mobile phase of the column consisted of $0.1 \mathrm{M}$ triethylamine (TEAA) pH 7.0 with (Buffer B) or without (Buffer A) 25\% acetonitrile. The gradient was created by mixing buffers $A$ and $\mathrm{B}$, which, were delivered to the column at a constant rate of $0.9 \mathrm{ml} / \mathrm{min}$ for $4 \mathrm{~min}$. Chromatograms were interpreted by looking for differences in the elution profiles of the samples. Typically the presence of a SNP, where present as a heterozygote, was indicated by the presence of an extra peak.

\section{Sequencing}

All amplicons, where differences in DHPLC traces were evident between samples, were sequenced after purification (QIAquick PCR Purification Kit; QIAGEN). The samples were prepared for sequencing using a ABI PRISM ${ }^{\circledR}$ BigDyeT v3.0 Terminator Cycle Sequencing Ready Reaction Kit, following the manufacturers' instructions. The samples were separated and analysed on an ABI 377 or ABI 3100 DNA sequencer (Perkin Elmer).

\section{NCBI database}

Four SNPs were found in the SNP database (http:// www.ncbi.nlm.nih.gov/SNP/index.html) under the following IDs: Int6b, rs993464; Int6c, rs1742; Int10a, rs1502018; Int10b, rs150217. All 10 SNPs were identified on genomic contig NT_031915 and the marker-marker distances calculated as follows (see Figure 1): Int6a-Int6b=7347 bp; Int6b-Int6c=1522 bp; Int6c - Int7 $a=15005$ bp; Int7a Int7 $b=614 \mathrm{bp} ; \quad$ Int7 $\mathrm{b}-\mathrm{Ex} 8=634 \mathrm{bp} ; \quad$ Ex8 - Int8a=63 bp; Int $8 \mathrm{a}-$ Int $8 \mathrm{~b}=1373 \mathrm{bp} ; \quad$ Int $8 \mathrm{~b}-$ Int $10 \mathrm{a}=13352 \mathrm{bp} ; \quad$ Int $10 \mathrm{a}-$ Int10b=94 bp; Int10b $-\operatorname{Int} 11=2581 \mathrm{bp}$.

\section{Genotyping}

Genomic DNA was isolated from cheek swabs or blood using standard laboratory protocols. ${ }^{24}$ Genotyping of the SNPs required the design of primers to introduce a restric- tion enzyme (REN) or fluorescently labelled allele specific PCR. The primers, annealing temperatures, and RENs used are listed in Table 1. For PCR $25 \mathrm{ng}$ of genomic DNA was used in $25 \mu \mathrm{l}$ with $0.3 \mu \mathrm{M}$ of each primer, $1.5 \mathrm{mM} \mathrm{MgCl}_{2}$, $100 \mu \mathrm{M}$ deoxynucleotide triphosphate (dNTP), $20 \mathrm{~mm}$ ammonium sulphate, $75 \mathrm{~mm}$ Tris- $\mathrm{HCl}$ (pH 8.8), 0.01\% (v/ v) Tween ${ }^{\mathbb{R}} 20$ and $0.5 \mathrm{U}$ Taq DNA polymerase (Applied Biotechnologies, Epsom, UK). Reactions were performed in a 96-well microtiter plate, with an initial denaturation step at $96^{\circ} \mathrm{C}$ for $85 \mathrm{~s}$ and 35 cycles of denaturation at $96^{\circ} \mathrm{C}$ (35 s), annealing (1 min), and extension at $72^{\circ} \mathrm{C}(1 \mathrm{~min})$ on a DNA Engine Tetrad thermocycler (MJ Research, Watertown, MA, USA). The final extension step was $10 \mathrm{~min}$ at $72^{\circ} \mathrm{C}$. A total of $12 \mu \mathrm{l}$ of the SNP reaction mixture was digested with $3 \mathrm{U}$ of the appropriate REN. The DNA fragments were separated by agarose gel electrophoresis and visualised by ethidium bromide fluorescence. Where partial digestion by the restriction enzyme was suspected, the digestion was repeated with three-fold more enzyme. Fluorescently labelled PCRs were visualised on either an ABI PRISM 377 Genetic Analyzer or an ABI PRISM 3100 Genetic Analyzer.

\section{Statistical Analysis}

Allele and genotype frequencies in the control and proband populations were compared by means of the $\chi^{2}$ test. The family-based analyses: $\mathrm{HHRR}^{25}$ and $\mathrm{TDT}^{26}$ were performed as described. Haplotype frequencies were estimated using the EM algorithm implemented in EHPLUS using the computed likelihood statistics for haplotype frequency comparisons between probands and controls. ${ }^{25,27}$ Linkage disequilibrium (LD) parameters $\mathrm{R}$ and $\mathrm{D}^{\prime}$ were calculated as described by Klitz et $a l^{28}$ using the computer program 2LD (www.iop.kcl.ac.uk/IoP/Departments/PsychMed/GEpiBSt/ software.stm). Empirical $P$ values ${ }^{29}$ were calculated by FASTEHPLUS. Logistic regression according to the method described by Cordell and Clayton ${ }^{30}$ was performed using the Stata statistical package.

\section{Results}

Each region to be screened for polymorphic variation was amplified from 30 unrelated individuals (60 chromosomes), which has $95 \%$ power to detect mutations of frequency $\geqslant 5 \%$. After confirmation by sequencing, ten novel SNPs of frequency $>5 \%$ were identified and genotyped in the case-control sample. All genotype frequencies were in Hardy-Weinberg equilibrium in both case and control samples (data not shown).

As shown in Table 2, four novel SNPs gave significant results by allele: Int6a, $P=0.0057$; Int7b, $P=0.00029$; Int8a, $P=0.0015$ and Int8b, $P=0.010$ with ORs between 1.5 and 2.4. Significant results were also obtained by genotype with $P$ values of $0.025,0.0015,0.0057$ and 0.029 respectively (data not shown). For comparison, results with an increased sample size for Ex8, the SNP in the original study, ${ }^{21}$ has 
Table 1 Positions, primers and detection methods of CACNA1A polymorphisms

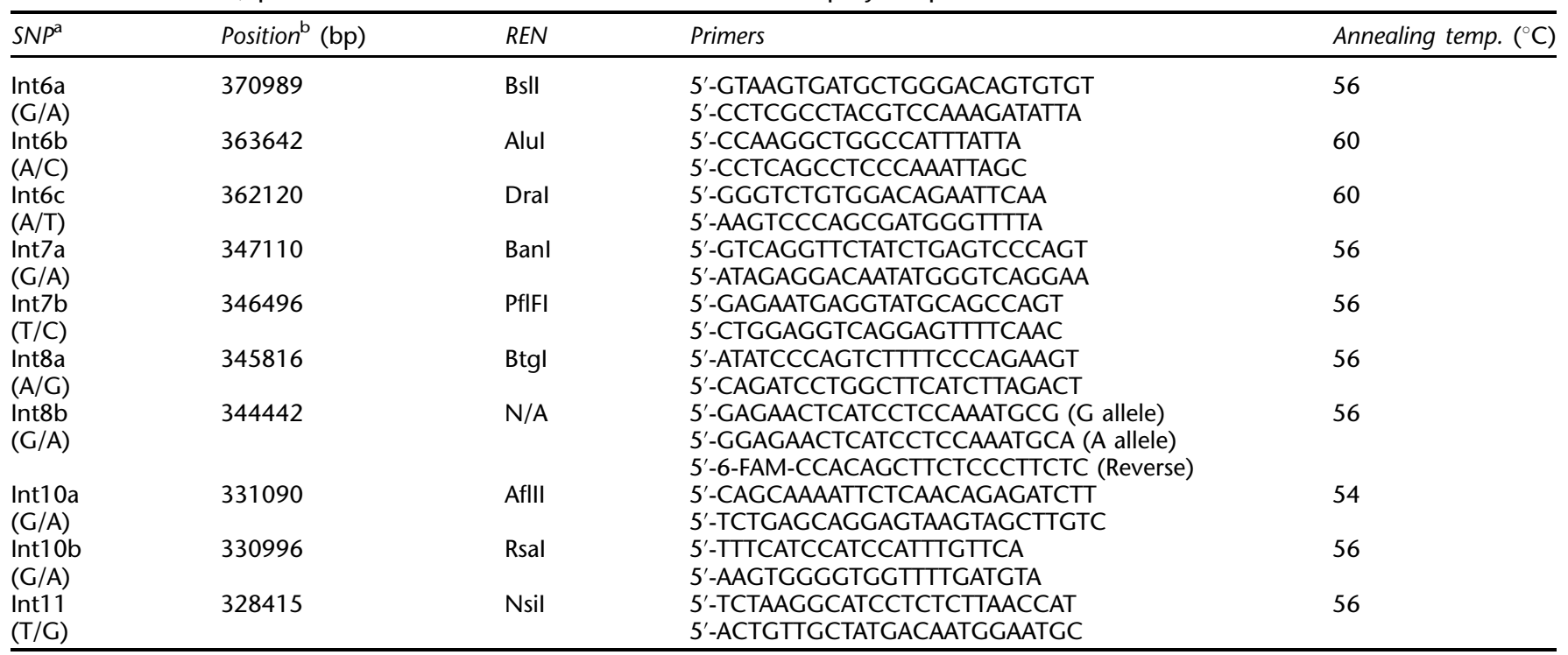

${ }^{\mathrm{a}}$ Major allele given first; ${ }^{\mathrm{b}}$ genomic contig, accession no. NT_031915.

Table 2 Allele frequencies in association studies with novel SNPS

\begin{tabular}{|c|c|c|c|c|c|}
\hline SNP & Probands/controls & Minor allele frequencies & Significance $^{\mathrm{a}}$ & Trios & Minor allele frequencies ${ }^{\mathrm{d}}$ \\
\hline Int6b & $200 / 231$ & $0.08 / 0.09$ & & & \\
\hline Int6c & $180 / 222$ & $0.08 / 0.11$ & & & \\
\hline Int7b & $223 / 232$ & $0.25 / 0.36$ & $\chi^{2}=13.14, P=0.00029, \mathrm{OR}=1.7(1.3-2.3)$ & 78 & $0.23 / 0.30$ \\
\hline Ex8 & $220 / 234$ & $0.26 / 0.36^{\mathrm{b}}$ & $\chi^{2}=10.57, P=0.0012, \mathrm{OR}=1.6(1.2-2.2)$ & 83 & $0.26 / 0.36^{c}$ \\
\hline Int8a & $217 / 233$ & $0.26 / 0.36$ & $\chi^{2}=10.07, P=0.0015, \mathrm{OR}=1.6(1.2-2.1)$ & 80 & $0.23 / 0.34$ \\
\hline Int10b & $160 / 224$ & $0.13 / 0.15$ & & & \\
\hline Int11 & $205 / 226$ & $0.24 / 0.23$ & & & \\
\hline
\end{tabular}

${ }^{a}$ Confidence intervals in parenthesis; ${ }^{b}$ increased sample size on earlier report; ${ }^{21}{ }^{c}$ same sample as earlier report, $\chi^{2}=4.07, P=0.044, O R=1.7(1.0-$ $2.7){ }^{21} \mathrm{~d}_{\text {transmitted/untransmitted. }}$

been included in Table 2. We further investigated SNPs Int6a, Int7b and Int8a in family trios. All three novel SNPs showed a distribution between transmitted and untransmitted alleles of similar magnitude and in the same direction as that seen between cases and controls, although, unlike Ex8, none reached statistical significance. Importantly, as with Ex8, there was no significant difference between the untransmitted and control allele frequencies, consistent with cases and controls having similar genetic backgrounds (data not shown). Similar results were obtained by TDT analysis (data not shown).

For the remaining six novel SNPs there were no significant differences between cases and controls either by allele frequency (Table 2) or genotype frequency (data not shown). Power calculations were made on the basis of detection of an association with odds ratio of 1.6 , the mean odds ratio of the four SNPs around exon 8 associated with IGE. These estimates show that of the SNPs not associated with IGE, Int10a and Int11 had $>80 \%$ power, Int $10 \mathrm{~b}$ $>70 \%$ power, Int7a $>60 \%$ power and Int6b and Int $6 \mathrm{c}$ $>50 \%$ power (data not shown).

For Ex8 and the four novel SNPs showing significant evidence of association, the odds ratios are consistent with either a small to moderate gene effect throughout the entire sample of IGE probands, or a larger effect limited to a subset. To address this issue we examined the association of the five SNPs in relation to the clinical data and found no increased association with any of the variables or ILAE syndromes. Table 3 shows the results for Int7b and pairs of mutually exclusive subsets and very similar allele frequencies are seen throughout. To test for heterogeneity among syndromes, we analysed these data as a 
multiple logistic regression in which the SNP allele was the dependent variable and the different phenotypes were binary predictor variables. Equating the phenotype-specific regression coefficients did not result in any significant reduction in fit (likelihood ratio $\chi^{2}=2.48,3$ d.f., $P=0.48$ ), indicating that there was no evidence that the specific syndrome was relevant to the association with Int7b. Similar results were obtained with the other four associated SNPs and by using the presence of each major seizure type as phenotype (data not shown).

Table 3 Allele frequencies of Int7b in different clinical subgroups

\begin{tabular}{|c|c|c|c|}
\hline $\begin{array}{l}\text { Patient } \\
\text { group }\end{array}$ & $T^{\mathrm{a}}$ & $C^{a}$ & Significance $^{\mathrm{b}}$ \\
\hline $\mathrm{IAE}^{\mathrm{C}}$ & $127(0.73)$ & $47(0.27)$ & $\chi^{2}=4.79, p=0.029, \mathrm{OR}=1.5$ \\
\hline Non-IAE & $139(0.79)$ & $37(0.21)$ & $\chi^{2}=13.51, p=0.00024, O R=2.1$ \\
\hline JME & $48(0.80)$ & $12(0.20)$ & $\chi^{2}=6.19, \mathrm{p}=0.013, \mathrm{OR}=2.3$ \\
\hline Non-JME & $218(0.75)$ & $72(0.25)$ & $\chi^{2}=10.65, p=0.0011, O R=1.7$ \\
\hline EGTCS $^{d}$ & $70(0.78)$ & $20(0.22)$ & $\chi^{2}=6.58, p=0.010, \mathrm{OR}=2.0$ \\
\hline Non-EGTCS & $196(0.75)$ & $64(0.25)$ & $\chi^{2}=10.28, p=0.0013, O R=1.7$ \\
\hline Controls & $296(0.64)$ & $168(0.36)$ & \\
\hline
\end{tabular}

We investigated LD between pairs of SNPs by analysis of the combined genotype data for cases and controls. We generated two measures of LD: the global statistic $\mathrm{R}$ and the statistic $\mathrm{D}^{\prime}$ that takes account of the limits on $\mathrm{R}$ imposed by different allele frequencies of the marker pair. The four SNPs around exon 8 associated with IGE (Int7b, Ex8, Int8a and Int8b) showed very strong LD with each other (Figure 1), having both high $\mathrm{R}$ and $\mathrm{D}^{\prime}$ values. This represents a region or block of associated markers, that we will refer to as Exon 8 Block (X8B). Extension of X8B in both directions showed reduced levels of LD. The other SNP that was significantly associated with IGE (Int6a) exhibited no LD with X8B (Figure 1).

A haplotype analysis of all the markers was performed using haplotype frequencies predicted by the $\mathrm{EH}$ programme and tested for association with IGE. Of the pairwise haplotypes within X8B, haplotypes of Int7b, Ex8 and Int8a each with Int8b were more significantly associated than either SNP alone (Figure 1). All pairwise haplotypes of Int6a and each SNP in X8B were similarly more significantly associated with IGE (Figure 1). Highly significant associations $\left(P<10^{-6}\right)$ were found for both the 3-marker and 5-marker haplotypes shown in Figure 1. (Because of uncertainty over degrees of freedom in the 5-marker haplotype, we used empirical $P$-value calculated by a permutation
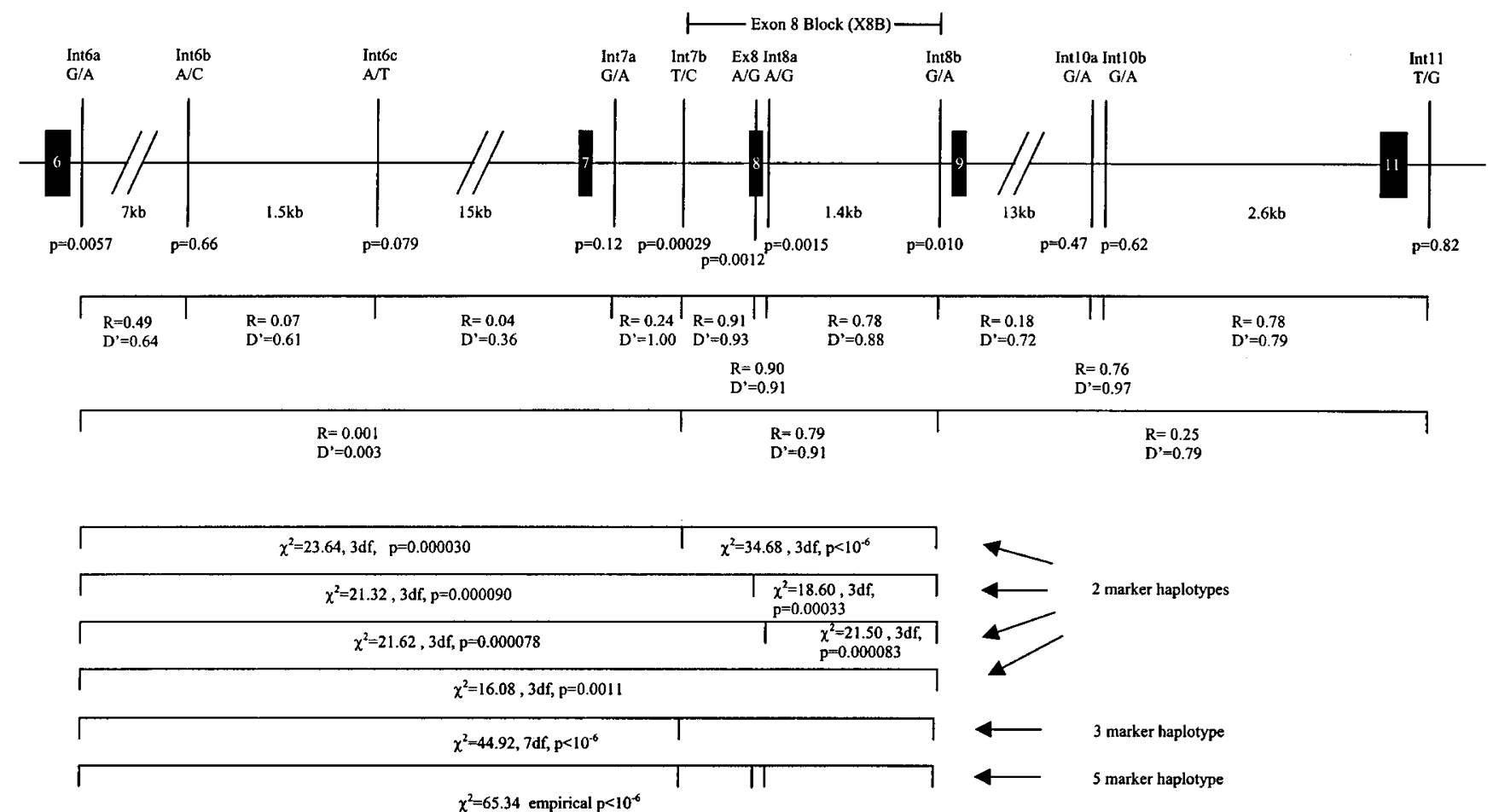

Figure 1 Map of CACNA1A between exons 6 and 11. Exons are shown by solid blocks. Distances between SNPs as indicated on the map. LD relationships between pairs of SNPs are shown immediately below the map with haplotypes between SNPs at the bottom. Significant $P$-values for case/control association studies are given for single marker allele frequencies (see Table 2 for details) and haplotype frequencies. 
procedure. $\left.{ }^{29}\right)$ Significant association with IGE $\left(\chi^{2}=15.60,3\right.$ d.f., $P=0.0014$ ) was also found for the predicted haplotypes of SNPs Int10a and Int11 (data not shown).

The contributions of the 5 SNPs associated with IGE were analysed by logistic regression. ${ }^{30}$ When five separate models each involving just one SNP were specified each one was significantly different from the null model and each SNP within the model had a significant odds ratio (data not shown). However, when the data were fitted to a single model involving all five SNPs $\left(\chi^{2}=22.57,5\right.$ d.f., $\left.P=0.0004\right)$, only Int6a and Int8a had odds ratios much greater than 1 , of which that for Int6a reached significance (Table 4). When a stepwise procedure was adopted the optimum model required only the same two SNPs, Int6a and Int8a $\left(\chi^{2}=21.98,2\right.$ d.f., $\left.P=0.000017\right)$. An identical optimum was achieved irrespective of whether increasing numbers of SNPs were progressively added or removed from a starting configuration with all five SNPs.

\section{Discussion}

We have previously reported a strong association between IGE and a SNP in exon 8 of CACNA1A both by case-control and within-family association studies. ${ }^{21}$ We have now identified several novel SNPs and, using an expanded version of the same sample, have shown that a total of five SNPs within a $30 \mathrm{~kb}$ region between exons 6 and 9 are associated with IGE. None of the associated SNPs have an obvious functional effect. Each association with IGE is therefore likely to be the result of linkage disequilibrium (LD) between the SNP and a putative functionally significant variant (FSV).

Recent data suggest that high levels of LD occur within blocks of SNP markers, that typically extend from a few $\mathrm{kb}$ to several hundred $\mathrm{kb}$, with a mean of around $60 \mathrm{~kb}^{31-33}$ Within these blocks, SNPs are closely associated with each other and it is in general impossible to differentiate the functional variant from the other SNPs using association data alone. These high LD regions appear to be separated by shorter regions of around 1-2 kb, in which higher recombination rates occur (recombination hotspots). This means that LD between blocks is reduced or even absent.

Table 4 Logistic regression using 5 SNP and stepwise models

\begin{tabular}{lllr}
\hline Model & SNP & \multicolumn{1}{l}{$\begin{array}{l}\text { Odds } \\
\text { ratio }^{\mathrm{a}}\end{array}$} & \multicolumn{1}{c}{$\begin{array}{l}\text { Wald } \\
\text { statistic }\end{array}$} \\
\hline 5 SNP model & Int6a & $2.61(1.30-5.26)$ & $2.68, P=0.01$ \\
& Int7b & $0.74(0.31-1.78)$ & $-0.66, P=0.51$ \\
& Ex8 & $1.14(0.52-2.50)$ & $0.31, P=0.75$ \\
& Int8a & $2.22(0.92-5.36)$ & $1.78, P=0.08$ \\
Stepwise model $^{\mathrm{b}}{ }^{\mathrm{N}}$ & Int8b & $0.95(0.55-1.64)$ & $-0.18, P=0.86$ \\
& Int6a & $2.61(1.30-5.27)$ & $2.69, P=0.01$ \\
& Int8a & $1.83(1.32-2.54)$ & $3.64, P=0.0003$ \\
\hline
\end{tabular}

${ }^{a}$ Confidence intervals in parenthesis; ${ }^{b}$ Identical figures were obtained for either forwards or backwards regression.
The data presented here identify a group of four SNPs in such a block spanning a region of $2 \mathrm{~kb}$ around exon 8 (X8B), which are all in strong LD with each other and all associated with IGE. Another SNP (Ex6a), approximately $25 \mathrm{~kb}$ distant on the $5^{\prime}$ side, is not in LD with X8B and yet is strongly associated with IGE. This finding is likely to result from either two FSVs in the same gene that are independently associated with these two groups of SNPs, or a single FSV in LD both with X8B and with Int6a.

If a single FSV were responsible for all five associations with IGE, it would have to be in LD with all five SNPs. This could occur if the FSV were located between Int6a and Int7b such that some LD between the FSV and each SNP has survived despite the loss of LD between the two SNPs. Some support for this scenario was obtained by examination of the six most common 5-marker haplotypes which account for around 95\% of the chromosomes in both cases and controls (data not shown). These haplotypes are consistent with a parsimonious model, where each haplotype is derived from two ancestral haplotypes, conferring increased risk of IGE (GTAAG) or protection (ACGGA). For all five SNPs IGE is associated with the major allele, suggesting that the risk ancestral haplotype was much more common than the protective ancestral haplotype. This could equally be due to a susceptibility FSV arising on the common ancestral haplotype or a protective FSV arising on the rarer ancestral haplotype.

Haplotype analysis of the five SNPs showed that pairwise haplotypes of Int6a with each of the four SNPs of X8B are more significantly associated with IGE than is either SNP alone (Figure 1) and highly significant associations were also found for three- and five-marker haplotypes. Furthermore, logistic regression demonstrated that no single SNP was able to explain the association of epilepsy with this gene, suggesting the possible involvement of two FSVs. However, none of these results can exclude the involvement of only one FSV in the association. Discrimination between these two alternatives will only be possible after identification and analysis of all SNPs within or close to this region of the gene.

Haplotype analysis of SNPs Int10a (G/A) and Int11 (T/G), which individually showed no association with IGE, showed significant differences between cases and controls. However, analysis of the four predicted haplotypes showed that GT and AG were apparently protective while GG and AT were both associated with increased risk (data not shown). As neither pair of risk or protective haplotypes share an allele in common, this result is inconsistent with an FSV occurring on a particular ancestral haplotype. This association was viewed as a likely false positive and not analysed further.

Some caution in the interpretation of all these data is necessary following a recent report by Sander et al, ${ }^{34}$ which failed to replicate our original association with the SNP Ex8. ${ }^{21}$ Their method of recruitment clearly differed from 
ours. Ours is an unselected broad-based IGE cohort with generalised spike/polyspike wave on EEG which was classified post recruitment. Theirs appears to consist of a grouping together of homogenous syndromes. JME, for example, constitutes $39 \%$ of their total population based sample compared to $17 \%$ in ours, although our finding was of significant association irrespective of clinical subtype of IGE. Their report does not mention how many patients had a family history for epilepsy but it is likely to be higher than in our sample as the same group has been part of a consortium for ascertaining cases with homogenous phenotypes for linkage studies. The presence in one cohort of a significant number of probands with a positive family history might be expected to result in a different range of variants being represented. Another possible explanation is that the two populations, collected from different European locations, differ in their genetic substructure (distribution of haplotypes) or in the balance of genetic risk factors (genetic heterogeneity). We have observed significant allele frequency differences between the two centres for other SNPs. ${ }^{35}$ While it remains possible that the findings reported here result from mere chance alone, this is highly unlikely given the lack of LD between one of the SNPs associated with IGE and the other four.

The work reported here and in our previous paper $^{21}$ has largely excluded the region to the $3^{\prime}$ of exon 10 . We have not yet excluded the region $5^{\prime}$ of exon 6 , but as discussed above the region of greatest interest lies between exons 6 and 9. We have screened exons $6-9$ by DHPLC analysis of genomic DNA from at least 30 IGE patients and 30 controls without finding any novel variant. We have also screened exons 1-13 of CACNA1A in cDNA derived from 16 controls with similar lack of success (data not shown). The putative susceptibility variant(s) in CACNA1A are therefore likely to be located in an intron and probably function either by affecting expression or by influencing splicing.

An alternatively spliced transcript of the rat homologue Cacna1a has been reported with differences at three sites. It expresses a channel with distinct properties that may be relevant to the different properties of P- and Q-type channels. ${ }^{36}$ One of these changes is at the exon 10 acceptor site which is only $2.6 \mathrm{~kb}$ from X8B associated with IGE. If similar alternative splicing occurs in humans it is conceivable that it might be modulated by an FSV. It is also possible that there is more alternative splicing yet to be discovered.

In humans several mutations in CACNA1A have been described which give rise to familial hemiplegic migraine (FHM), episodic ataxia type 2 (EA-2) and spinocerebellar ataxia type 6 (SCA6). ${ }^{19,37}$ Fifteen different amino acid substitutions at positions throughout the calcium channel subunit have been identified in FHM families, ${ }^{38}$ one of which is in the P loop of the second repeat and very close to the position of the tottering mouse mutation. ${ }^{18}$ Patients with this mutation also have progressive ataxia associated with FHM, as have patients with some of the other amino acid substitutions. ${ }^{38,39}$ Fourteen mutations in EA-2 families have also been described. ${ }^{37}$

Recently, an EA-2 patient was described who also has an absence epilepsy. ${ }^{20}$ The mutation in this patient results in a premature stop in the S6 transmembrane domain of the fourth repeat preventing translation of the C-terminal intracellular region. We have investigated this mutation in our cohort of IGE patients but failed to detect it. ${ }^{40}$ Since this mutation is rare it is unlikely to influence risk for common forms of IGE and cannot explain the data presented here. It is interesting that this mutation and the mutation in the tottering mouse are both implicated in absences, yet we could find no evidence for the association between CACNA1A SNPs and IGE being specific for absence epilepsies. This may be due to a similarly altered function in the two absence-causing mutations, whereas the FSV(s) responsible for the association with IGE may affect expression of the gene.

In conclusion, our data support one or two intronic FSV(s), close to the region between exons 6 and 9 of CACNA1A, that predispose to IGE but are not specific to any syndrome. We are currently screening for more SNPs in this critical region of the gene for fine mapping association studies. We are also investigating CACNA1A mRNA from the IOP brain bank to identify possible functional effects of the FSV.

\section{Acknowledgements}

We would like to thank Jackie Blower and Corrine Wilkins for their hard work with the sample collection and the MRC London Brain Bank at the IOP for the use of post-mortem brain material for mutation detection in exonic regions of the gene. We would like to thank Dr D McCormick in collecting paediatric patients and Shaun Purcell for his assistance with some of the statistics. We would like to acknowledge the support of the Epilepsy Research Foundation for funding the initial sample collection and for funding B Suarez-Merino in some of the mutation detection work. We would also like to thank King's JRC for supporting B Chioza, the MRC for a studentship to $H$ Wilkie, the Fund for Epilepsy for supporting A Osei-Lah, and Internal Project Grant Scheme R\&D East Kent Hospitals NHS Trust.

\section{References}

1 Beck-Mannagetta G, Janz D, Hoffmeister U: Genetics of the Epilepsies. Berlin: Springer-Verlag, 1989; pp 119-126.

2 Gedda L, Tatarelli R: Essential isochronic epilepsy in MZ twin pairs. Acta Genet Med Gemellol (Roma) 1971; 20: 380-383.

3 Berkovic SF, Howell RA, Hay DA, Hopper JL: Epilepsies in twins: genetics of the major epilepsy syndromes. Ann Neurol 1998; 43: $435-445$.

4 Commission on Classification Terminology of the International League Against Epilepsy: Proposal for revised classification of epilepsies and epileptic syndromes. Epilepsia 1989; 30: 389-399.

5 Beck-Mannagetta G, Janz D: Syndrome-related genetics in generalized epilepsy. Epilepsy Res 1991; 4 (Suppl): 105-111.

6 Bianchi A: Study of concordance of symptoms in families with absence epilepsies; In Panayiotopoulos D (ed): Typical absences and related epileptic syndromes. Europe: Churchill Communications Limited, 1995, pp 328-337. 
7 Berkovic SF, Howell RA, Hay DA, Hopper JL: Epilepsies in twins. London: John Libbey, 1994; pp 157-164.

8 Risch N, Merikangas K: The future of genetic studies of complex human diseases. Science 1996; 273: 1516-1517.

9 Risch NJ: Searching for genetic determinants in the new millennium. Nature 2000; 405: 847-856.

10 Heller AH, Dichter MA, Sidman RL: Anticonvulsant sensitivity of absence seizures in the tottering mutant mouse. Epilepsia 1983; 24: $25-34$.

11 de Curtis M, Avanzini G: Thalamic regulation of epileptic spike and wave discharges. Funct Neurol 1994; 9: 307-326.

12 Snead III OC: Basic mechanisms of generalized absence seizures. Ann Neurol 1995; 37: 146-157.

13 Fletcher CF, Lutz CM, O'Sullivan TN et al: Absence epilepsy in tottering mutant mice is associated with calcium channel defects. Cell 1996; 87: 607-617.

14 Burgess DL, Jones JM, Meisler MH, Noebels JL: Mutation of the $\mathrm{Ca} 2+$ channel beta subunit gene Cchb4 is associated with ataxia and seizures in the lethargic (lh) mouse. Cell 1997; 88: 385-392.

15 Barclay J, Balaguero N, Mione M et al: Ducky mouse phenotype of epilepsy and ataxia is associated with mutations in the Cacna2d2 gene and decreased calcium channel current in cerebellar Purkinje cells. J Neurosci 2001; 21: 6095-6104.

16 Mori Y, Wakamori M, Oda $S$ et al: Reduced voltage sensitivity of activation of $\mathrm{P} / \mathrm{Q}$-type $\mathrm{Ca} 2+$ channels is associated with the ataxic mouse mutation rolling Nagoya (tg(rol)). J Neurosci 2000; 20: $5654-5662$

17 Zwingman TA, Neumann PE, Noebels JL, Herrup K: Rocker is a new variant of the voltage-dependent calcium channel gene Cacna1a. J Neurosci 2001; 21: 1169-1178.

18 Ophoff RA, Terwindt GM, Vergouwe MN et al: Familial hemiplegic migraine and episodic ataxia type- 2 are caused by mutations in the Ca2+ channel gene CACNL1A4. Cell 1996; 87: 543-552.

19 Zhuchenko O, Bailey J, Bonnen P et al: Autosomal dominant cerebellar ataxia (SCA6) associated with small polyglutamine expansions in the alpha 1A-voltage-dependent calcium channel. Nat Genet 1997; 15: 62-69.

20 Jouvenceau A, Eunson LH, Spauschus A et al: Human epilepsy associated with dysfunction of the brain P/Q-type calcium channel. Lancet 2001; 358: 801-807.

21 Chioza B, Wilkie H, Nashef L et al: Association between the alpha (1a) calcium channel gene CACNA1A and idiopathic generalized epilepsy. Neurology 2001; 56: $1245-1246$.

22 Sander T, Peters C, Janz D et al: The gene encoding the alpha1Avoltage-dependent calcium channel (CACN1A4) is not a candidate for causing common subtypes of idiopathic generalized epilepsy. Epilepsy Res 1998; 29: 115-122.

23 Engel Jr J: A proposed diagnostic scheme for people with epileptic seizures and with epilepsy: report of the ILAE Task Force on Classification and Terminology. Epilepsia 2001; 42: 796-803.

24 Meulenbelt I, Droog S, Trommelen GJ, Boomsma DI, Slagboom PE: High-yield noninvasive human genomic DNA isolation method for genetic studies in geographically dispersed families and populations. Am J Hum Genet 1995; 57: 1252-1254.
25 Terwilliger J, Ott J: Handbook of Human Genetic Linkage. Baltimore: The Johns Hopkins University Press, 1994; pp 188-198.

26 Spielman RS, McGinnis RE, Ewens WJ: Transmission test for linkage disequilibrium: the insulin gene region and insulindependent diabetes mellitus (IDDM). Am J Hum Genet 1993; 52 $506-516$.

27 Xie X, Ott J: Testing linkage disequilibrium between a disease gene and marker loci. Am J Hum Genet 1993; 53 (Suppl): 1107.

28 Klitz W, Stephens JC, Grote M, Carrington M: Discordant patterns of linkage disequilibrium of the peptide-transporter loci within the HLA class II region. Am J Hum Genet 1995; 57: 1436-1444.

29 Zhao JH, Curtis D, Sham PC: Model-free analysis and permutation tests for allelic associations. Hum Hered 2000; 50: 133-139.

30 Cordell HJ, Clayton DG: A unified stepwise regression procedure for evaluating the relative effects of polymorphisms within a gene using case/control or family data: application to HLA in type 1 diabetes. Am J Hum Genet 2002; 70: 124-141.

31 Daly MJ, Rioux JD, Schaffner SF, Hudson TJ, Lander ES: High-resolution haplotype structure in the human genome. Nat Genet 2001; 29: 229-232.

32 Johnson GC, Esposito L, Barratt BJ et al: Haplotype tagging for the identification of common disease genes. Nat Genet 2001; 29 : $233-237$.

33 Reich DE, Cargill M, Bolk S et al: Linkage disequilibrium in the human genome. Nature 2001; 411: 199-204.

34 Sander T, Toliat M, Heils A, Becker C, Nurnberg P: Failure to replicate an allelic association between an exon 8 polymorphism of the human alpha1A calcium channel gene and common syndromes of idiopathic generalized epilepsy. Epilepsy Res 2002; 49: $173-177$.

35 Wilkie HJ, Osei-Lah A, Chioza B et al: Association of the muopioid receceptor subunit gene and idiopathic generalised epilepsy. Neurology 2002; 59: 724-728.

36 Bourinet E, Soong TW, Sutton K et al: Splicing of alpha 1A subunit gene generates phenotypic variants of P- and Q-type calcium channels. Nat Neurosci 1999; 2: 407-415.

37 Ophoff RA, Terwindt GM, Frants RR, Ferrari MD: P/Q-type Ca2+ channel defects in migraine, ataxia and epilepsy. Trends Pharmacol Sci 1998; 19: 121-127.

38 Wada T, Kobayashi N, Takahashi Y, Aoki T, Watanabe T, Saitoh S: Wide clinical variability in a family with a CACNA1A T666m mutation: hemiplegic migraine, coma, and progressive ataxia. Pediatr Neurol 2002; 26: 47-50.

39 Ducros A, Denier C, Joutel A et al: Recurrence of the T666M calcium channel CACNA1A gene mutation in familial hemiplegic migraine with progressive cerebellar ataxia. Am J Hum Genet 1999; 64: 89-98.

40 Chioza B, Nashef L, Asherson P, Makoff AJ: CACNA1A and P/Qtype calcium channels in idiopathic generalised epilepsy. Lancet 2002; 359: 258. 